\title{
A Mathematical Analysis of Glacier Surges
}

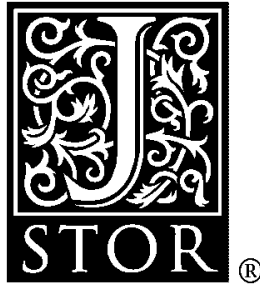

\author{
A. C. Fowler
}

SIAM Journal on Applied Mathematics, Vol. 49, No. 1. (Feb., 1989), pp. 246-263.

Stable URL:

http://links.jstor.org/sici?sici=0036-1399\%28198902\%2949\%3A1\%3C246\%3AAMAOGS\%3E2.0.CO\%3B2-4

SIAM Journal on Applied Mathematics is currently published by Society for Industrial and Applied Mathematics.

Your use of the JSTOR archive indicates your acceptance of JSTOR's Terms and Conditions of Use, available at

http://www.jstor.org/about/terms.html. JSTOR's Terms and Conditions of Use provides, in part, that unless you have obtained prior permission, you may not download an entire issue of a journal or multiple copies of articles, and you may use content in the JSTOR archive only for your personal, non-commercial use.

Please contact the publisher regarding any further use of this work. Publisher contact information may be obtained at http://www.jstor.org/journals/siam.html.

Each copy of any part of a JSTOR transmission must contain the same copyright notice that appears on the screen or printed page of such transmission.

The JSTOR Archive is a trusted digital repository providing for long-term preservation and access to leading academic journals and scholarly literature from around the world. The Archive is supported by libraries, scholarly societies, publishers, and foundations. It is an initiative of JSTOR, a not-for-profit organization with a mission to help the scholarly community take advantage of advances in technology. For more information regarding JSTOR, please contact support@jstor.org. 


\title{
A MATHEMATICAL ANALYSIS OF GLACIER SURGES*
}

\author{
A. C. FOWLER $\dagger$
}

\begin{abstract}
This paper describes the phenomena of glacier surges and presents a mathematical model based on realistic descriptions of glacier physics, which purports to describe the main features of the flow. The analysis reveals and predicts a variety of phenomena, many of which have been observed to occur, and gives explicit estimates for such quantities as surge front extent, "mini-surge" propagation speed, and oscillation period.
\end{abstract}

Key words. glacier surges, relaxation oscillations, asymptotic methods

AMS(MOS) subject classifications. 86A05, 35B25

1. Introduction. Glacier surges are a phenomenon of some interest, as the recent (1986) surge of the Hubbard Glacier in Alaska indicates. A surge is a sudden, rapid advance of a glacier beyond its normal "equilibrium" position. Following a surge, during which ice velocities can increase by factors of about one hundred, there is a slow, quiescent phase during which the glacier retreats: surging is thus essentially a periodic phenomena, and as such can be viewed as a relaxation oscillation.

The ability to make a useful model for glacier surges rests on having a sound description of the physics involved. A significant step in this direction was an excellent field study on the Variegated Glacier, also in Alaska, and close to the Hubbard, by Kamb et al. [12]. Pre- and post-surge aerial photographs of this glacier are shown in Figs. 1(a) and (b). To give some idea of scale, the Variegated is around $20 \mathrm{~km}$ long, $1 \mathrm{~km}$ wide, and $400 \mathrm{~m}$ deep. During its surges, it advances several kilometres at velocities of up to about fifty metres $a$ day (compared to typical quiescent velocities of around a hundred metres a year).

It is thought that this exceptional velocity is due to sliding at the base. In a quiescent phase, glaciers move by shearing (due to solid state creep processes) and also by sliding over a lubricated, but bumpy, bed. Glacier sliding occurs if the ice is temperate (at the melting point), as is the case for the Variegated, for example.

When a glacier is temperate, there is a subglacial hydraulic system which carries water towards the glacier snout. The pressure in this hydraulic system is not necessarily equal to the overburden ice pressure and is determined by a balance between tunnel closure and meltback (Röthlisberger [21]). A balance of these two processes leads to a theoretical prediction of basal water pressure which is at least comparable to observations, although a completely satisfactory quantitative agreement is not obtained (Iken and Bindschadler [11]).

The sliding velocity at the glacier base is considered to depend on both the shear stress $\tau$ and the basal water pressure $p_{w}$. More specifically, the latter dependence is usually assumed to be on the effective pressure $N=p_{i}-p_{w}$, where $p_{i}$ is the ice overburden pressure. Then we should physically expect that $u$ increases with applied stress $\tau$, and decreases with $N$; that is, if $\tau=\tau(u, N)$, then $\partial \tau / \partial u>0, \partial \tau / \partial N>0$. Theoretical "sliding laws" (Nye [18], Lliboutry [16], and Fowler [7]) and field velocity measurements (Bindschadler [1], Iken and Bindschadler [11]) are consistent with this

* Received by the editors September 16, 1987; accepted for publication (in revised form) December $12,1987$.

† Mathematical Institute, Oxford University, 24-29 St. Giles', Oxford OX1 3LB, United Kingdom. 
(a)

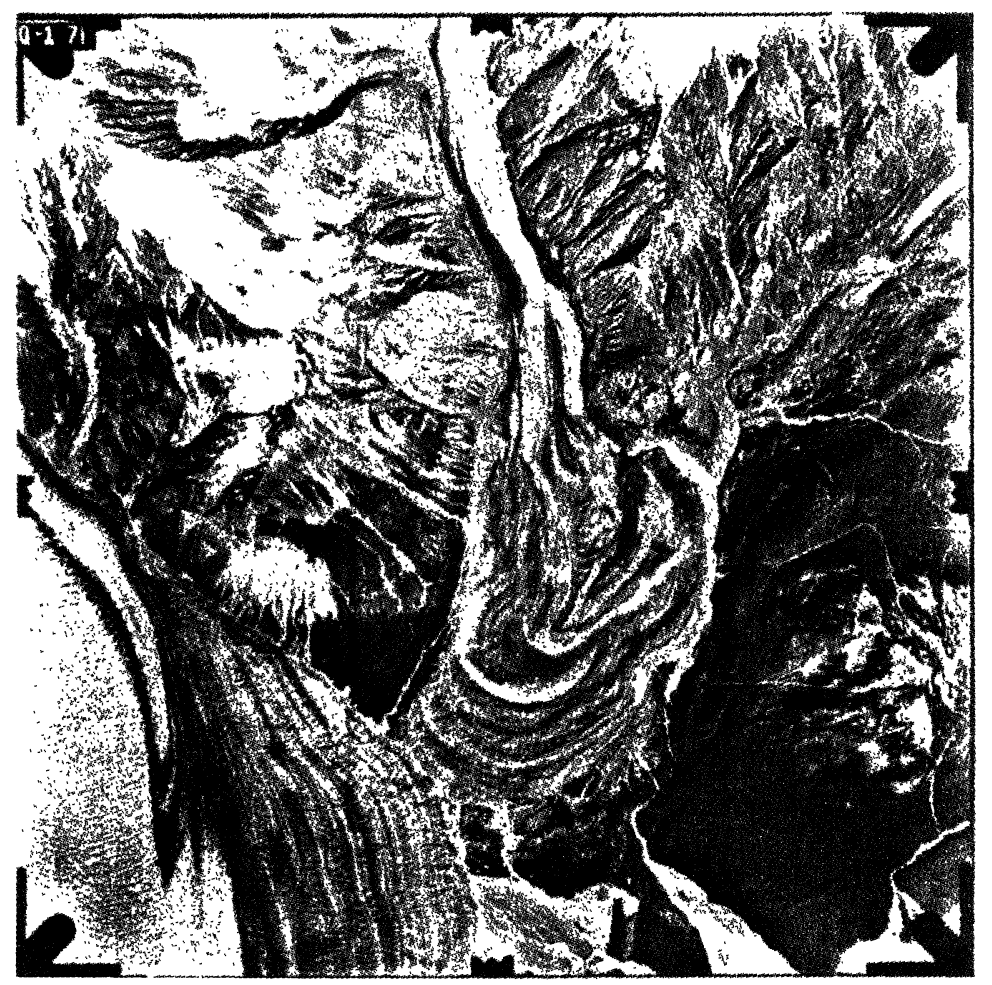

(b)

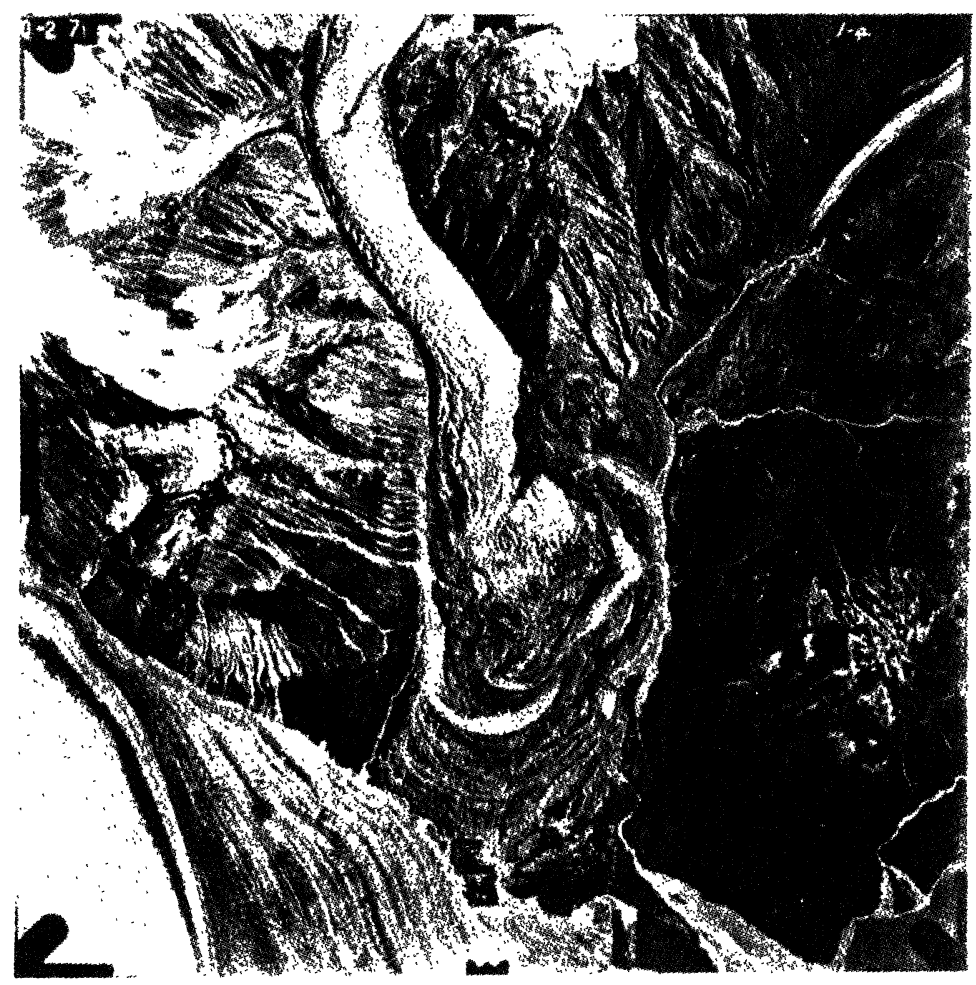

FiG. 1(a), (b). Pre- and post-surge aerial photographs of Variegated Glacier, Alaska. These views were taken in July 1982 and August 1983, respectively, between which time the glacier has advanced several kilometres. Photographs are by courtesy of North Pacific Aerial Surveys, Inc., Anchorage, Alaska. 
viewpoint. However, it should be mentioned that for such a sliding law, we would typically expect sliding velocity to vary synchronously with basal water pressure, and in the same sense, but this is not necessarily the case (Paterson [20]).

The most significant new observation made by Kamb et al. [12] was of a fundamental change in the drainage characteristics of the Variegated Glacier as it surged. During the surge, the basal water pressures were much higher (in keeping with the sliding law referred to above), and by means of dye-tracer injection studies, it was inferred that during these periods of high pressure, the flow velocity was much smaller. The suggestion is that during the surge, most water drains through the cavities, the tunnel system essentially shutting down. Observations on Variegated Glacier strongly support the idea that this is in fact what happens. Theoretical descriptions of the drainage through such a network of linked cavities (Kamb [13], Kamb et al. [12], Walder [22], and Fowler [7]) support the view that water pressure is higher in such a drainage system, and furthermore suggest that drainage through linked cavities only is unstable to formation of a tunnel-drainage system if $u$ is small enough (Kamb), and that, conversely, a combined tunnel-cavity system is unstable to collapse of tunnels if $u$ is large enough (Fowler). The models are different, but presumably the physical processes are the same, and it seems reasonable that these various instabilities occur at the same sliding velocity.

2. Mathematical model. We consider the flow of a "shallow" glacier (depth « length) down a valley in the $x$ direction. We suppose the flow is predominantly by sliding, so that $u=u(x, t)$ represents the downstream flow. A dimensionless set of model equations has been derived by Fowler [6] (see also [5]):

$$
\begin{aligned}
& H_{t}+(u H)_{x}=s^{\prime}(x), \\
& H\left[1-\mu H_{x}\right]+\alpha\left[H u_{x}\left|u_{x}\right|^{(1 / n)-1}\right]_{x}=N f(\Lambda), \\
& \varepsilon N_{t}+N=g(\Lambda), \\
& \Lambda=u / N^{n} .
\end{aligned}
$$

In these equations $H$ is the glacier thickness, $u$ is the velocity, and $N$ is the effective pressure. The first represents conservation of mass, where the source term $s^{\prime}(x)$ represents accumulation or ablation at the glacier surface. The left-hand side of $(2.1 \mathrm{~b})$ is the basal shear stress, $\tau$. The expression is composed of three parts: $\tau \approx H$ represents the fundamental balance between the downslope force due to gravity, and the resistive shear stress: it is just a force balance for slab flow. The terms in $\mu$ and $\alpha$ are small corrections, both well known in glaciology. The $\mu$ term represents the effect of deviations of the surface from a parallel flow; an additional term representing basal topography has been suppressed, equivalent to assuming the base is $y=0$. The term in $\alpha$ represents a correction due to longitudinal stresses, which will be significant if velocity gradients are large (Paterson [19, p. 100]). Typical estimates for $\alpha$ and $\mu$ are $\alpha \sim 10^{-2}, \mu \sim 10^{-1}$ (Fowler [6]).

The equation (2.1b) is in fact the sliding law, that is, $\tau=N f\left(u / N^{n}\right)$. This form arises from theoretical reasons (Lliboutry [16], Fowler [7]). Consequently, the drainage characteristics really depend on $\Lambda=u / N^{n}$. As discussed in $\S 1$, for $\Lambda>\Lambda_{c}$ (a critical value), tunnel drainage is unstable and linked cavities are stable, whereas (we suppose) the opposite is the case for $\Lambda<\Lambda_{c}$. In equilibrium, the drainage characteristics would be given by $N=g(\Lambda)$, where for $\Lambda<\Lambda_{c}, g \approx N_{R}$, and for $\Lambda>\Lambda_{c}, g \approx N_{K}<N_{R}$. We 
denote the ratio by

$$
\delta=N_{K} / N_{R}
$$

The semi-empirical term in $\varepsilon$ is inserted to indicate that there is of course a relaxation time in passing from one drainage system to another. This time, $t_{\text {drain }}$, is the time scale for flooding the bed from a pre-existing tunnel, or for converting a linked-cavity system to a tunnel system. Then $\varepsilon$ is the ratio $t_{\text {drain }} / t_{\text {conv }}$, where the convective time scale $t_{\text {conv }}$ is of the order of 20 years or more.

Fowler [6] estimated $\varepsilon \sim 10^{-5}$, on the basis of a tunnel collapse time (by viscous closure) of more than one day. This is possibly rash, as the flooding of the bed would lead to a longer time scale, possibly $t_{\text {drain }} \sim$ one month, and then $\varepsilon \sim 10^{-2}$ or so. A word of caution is necessary here and will be repeated at intervals. We are seeking to understand the large scale features of surges, in particular the periodicity and the fast and slow phases. Such a relaxation oscillator will interact in a complicated way with time-varying inputs on a seasonal basis, such as annual variation in water production. We are not specifically concerned with such short-term variability, since it is considered peripheral to the main purpose. Equally, our model for the drainage characteristics is simplistic and neglects such effects as subglacial water storage and winter initiation of surges. Consequently, the model lacks realism in this respect and cannot be expected to simulate such features as "mini-surges" (Kamb et al. [12]); nevertheless, it is suggested that the present model can serve as a useful basis to which further complications can be added.

For the sake of completeness, let us summarize what the nature of these complications may be, in regard to the drainage system. There are at least four possible time scales governing the adjustment of basal drainage conditions to changes in water supply.

(i) The convective time scale for propagation of kinematic waves along tunnels (waves of perturbed cross section): with length $\sim 10^{4} \mathrm{~m}$, velocity $\sim 1 \mathrm{~m} \mathrm{~s}^{-1}$, this is $10^{4} \mathrm{~s} \sim$ three hours. However, in practice this may be irrelevant, since propagation of changes in $S$ (cross section) requires deformation of ice on the slower viscous closure time scale.

(ii) Viscous closure time scale. This is about a day, although it depends on what choice of flow law constant is appropriate.

(iii) Bed leakage time scale. As discussed above, this may be about one month.

(iv) Cavity adjustment time scale. The importance of transient cavity behaviour was emphasized by Iken [10]. The relevant time scale is the convective one. For bumps of dimension $10 \mathrm{~m}$, sliding rate $100 \mathrm{~m} y^{-1}$, this is also about one month.

Only when a basic hydrological model represents all these processes can one confidently go looking for explanations (in this model) of mini-surges, short-term "events," and so on.

There have been various more or less ad hoc models to explain surging behaviour. Prominent among these is the paper by Budd [2], whose model may be compared with that presented here. In a sense, the approach is the same. Conservation of mass is invoked, the definition of the basal shear stress is the same, and only the sliding law is very different. Budd's "sliding law" (his equation (14)) is entirely empirical, and in fact not a local sliding law as such. More reasonable might have been a multivalued sliding law $\tau=f(u)$ as proposed by Lliboutry [15], but a numerical model for that would have failed, unless some relaxation mechanism for transition from slow to fast velocities were present. Although Röthlisberger's [21] hydraulic theory had been published, the crucial switching behaviour of the drainage system was not observed until the Variegated surge, and in the present model, this provides the relaxation 
mechanism (the term in $\varepsilon$ in (2.1c)), which is itself crucial: if we put $\varepsilon=0$, then (2.1) does not have continuous solutions, and a straightforward numerical approach will fail.

3. Analysis. There are some theoretical reasons (Fowler [7]) for adopting the form

$$
f(\Lambda)=c \Lambda^{1 / m}, \quad m>n,
$$

for the sliding law in (2.1b); at any rate, the schematic forms of $f$ and $g$ versus $\Lambda$ are as shown in Fig. 2(a) and (b). Where necessary, we will assume (3.1) for $f$, and a step function for $g$, as indicated before (2.2). The parameters $\alpha, \varepsilon$, and $\mu$ in (2.1) are all small, and it makes sense, to leading order, to ignore them. Then

$$
N \approx g(\Lambda), \quad H \approx N f(\Lambda), \quad \Lambda=u / N^{n},
$$

and it is not difficult to see that elimination of $N$ gives a multivalued relation between $H$ and $u$, as shown in Fig. 3. The resultant relationship between $Q=u H$ and $H$ is thus also multivalued, and is shown in Fig. 4. For the choice of functions $f$ and $g$ above, the sliding law in Fig. 3 is given by

$$
\begin{aligned}
& H \approx c N_{R}^{(m-n) / m} u^{1 / m}, \quad u<\Lambda_{c} N_{R}^{n}, \\
& H \approx c \delta^{(m-n) / m} N_{R}^{(m-n) / m} u^{1 / m}, \quad u>\delta^{n} \Lambda_{c} N_{R}^{n},
\end{aligned}
$$

these being connected by the unstable transition branch, $u \approx \Lambda_{c} N^{n}$.

The description of a surge cycle has been given by Fowler [6], and is briefly summarized in Fig. 5. Here the aim is to exhibit the mathematical detail necessary to cement the constituents of this picture together. We denote the two transition values

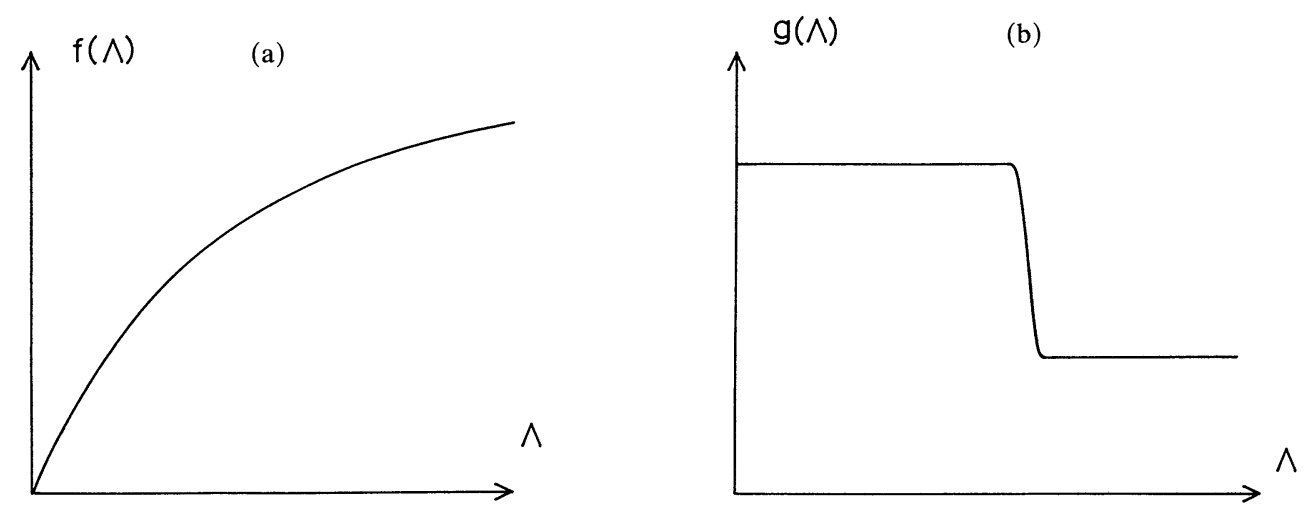

FIG. 2(a), (b). Schematic illustration of the sliding and drainage functions $f(\Lambda), g(\Lambda)$ (see text).

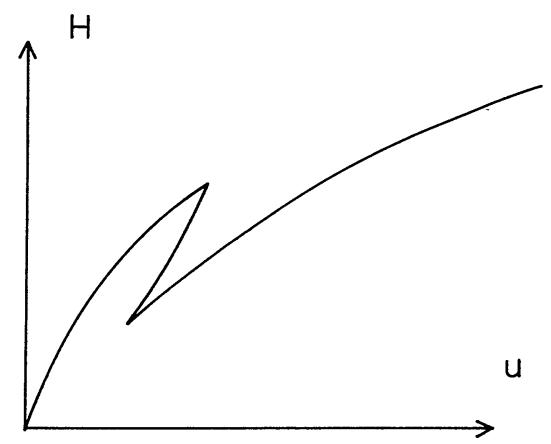

FIG. 3. $H$ as a multivalued function of $u$. 


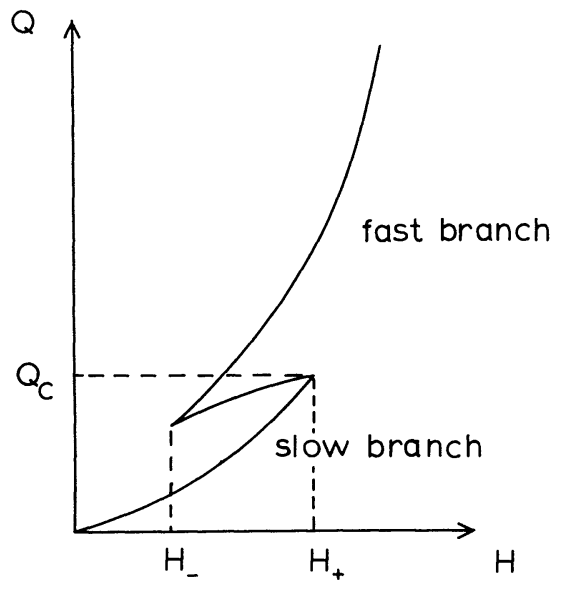

FIG. 4. $Q$ as a multivalued function of $H$.

of $H$ in Fig. 4 as $H_{+}$and $H_{-}$. The equilibrium profile of a glacier (solve (2.1a) for $Q$ with $\partial H / \partial t=0)$ is just $Q=s(x)$, so that, neglecting $\alpha$ and $\mu$, we have $H$ given from $Q(H)=s(x), Q(H)$ being given from Fig. 4. Now if the lower branch value of $Q$ at $H_{+}$, say $Q_{c}$, is less than the maximum value of the balance, $s_{\max }$, then an initial profile such as Fig. 5(a) will relax towards an intended "equilibrium" with $Q>Q_{\mathrm{c}}$. When the maximum value of $H, H_{\max }$, reaches $H_{+}$, this slow adjustment cannot continue, and neither can the reduced model: at least one of the parameters $\alpha, \mu, \varepsilon$, must become important in small scale transition regions. We argue below that in this activation stage, two activation waves propagate rapidly up and down glacier, and in so doing transfer the part of the glacier with $H_{-}<H<H_{+}$from the lower branch to the upper branch (see Fig. 5(b)). At the end of this activation stage, the central portion of the glacier is activated, moving at high velocities, and ready to slump (Fig. 5(c)). We thus need to show that rapidly propagating activation waves of the type described above exist, and that they can travel in either direction. These waves demarcate the moving boundary between the activated (linked-cavity) drainage system and the quiescent (tunnel) system. If $\delta$ in (3.3) is small enough, the upper branch has much higher velocity than the lower branch, and the activated region will slump forward rapidly (Fig. 5(d)) towards a new quasi-equilibrium. Mass balance is irrelevant to this state (Fig. 5(e)), which is thus one of constant $Q$ (hence $H \approx H_{-}$in our model). The rapid propagation of the surge front requires a shock structure analysis. In particular, Kamb et al. [12] observed an oscillatory decay behind the shock (alternating tensile/compressive zones), which we would like to explain. At the end of the surge, we then expect deactivation waves to propagate inwards from the boundaries, and the glacier resumes its pre-surge quiescent build-up.

Our purpose is then the following: taking the model (2.1) as given, we will first show that activation waves, deactivation waves, and tensile/compressive surge fronts exist and have characteristics at least comparable to observations on Variegated Glacier. In $\S 4$ we will formulate a set of surge criteria, based on this model, and see whether they compare reasonably to surging glaciers' characteristics, and also whether periods, etc., are of comparable size. We also mention some of the problems which may be of quantitative importance in this analysis.

(i) Activation waves. We seek a traveling wave solution which connects an activated region $\left(N=\delta N_{R}\right)$ to a quiescent one $\left(N=N_{R}\right)$. We expect $H$ to be approximately 


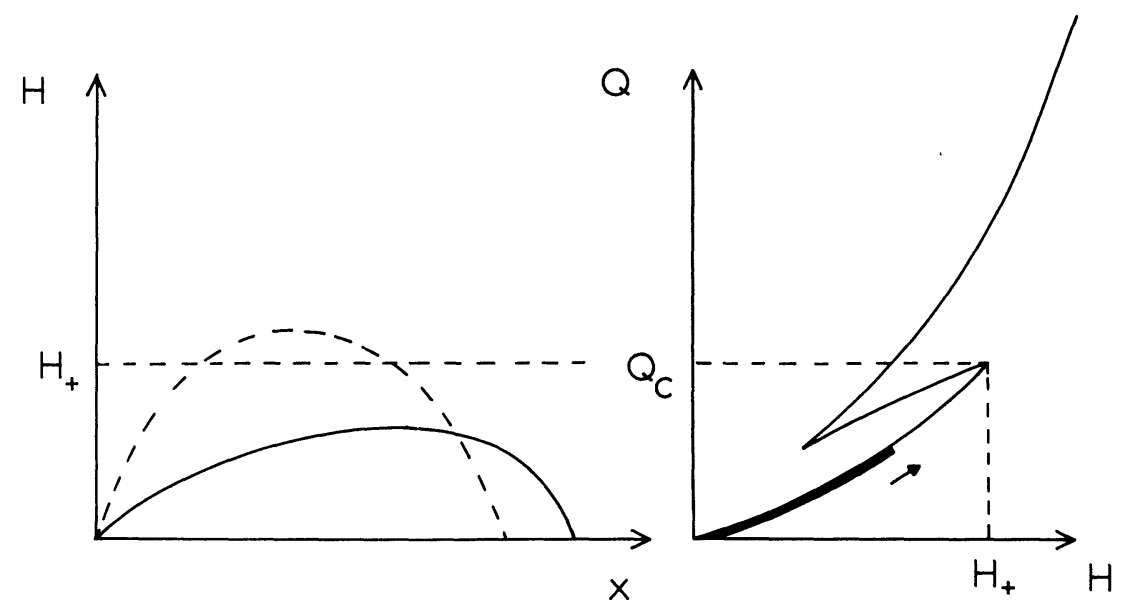

(a)

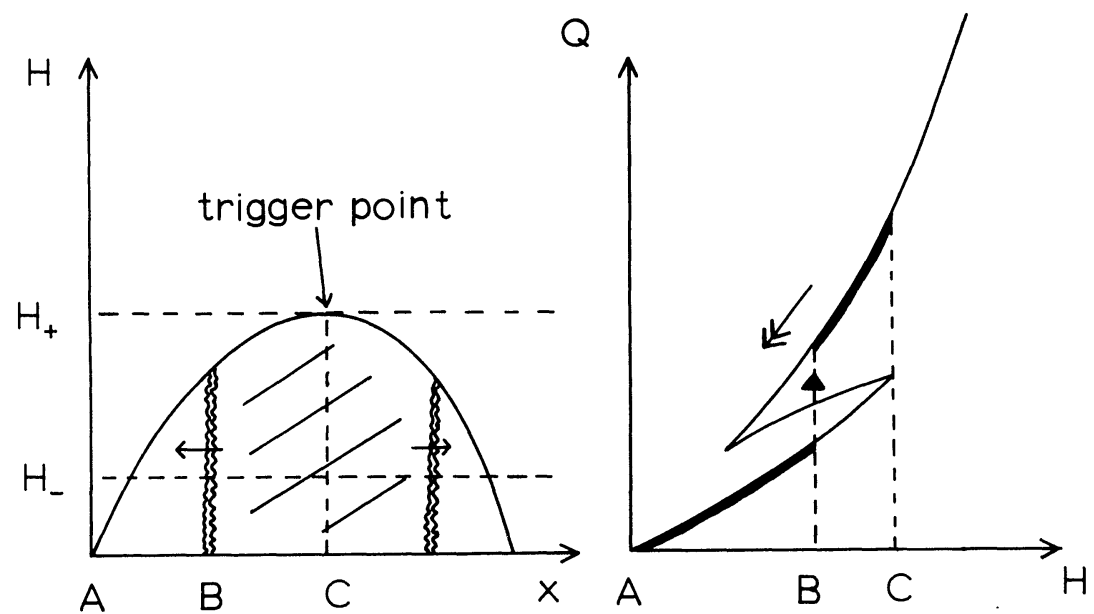

(b)

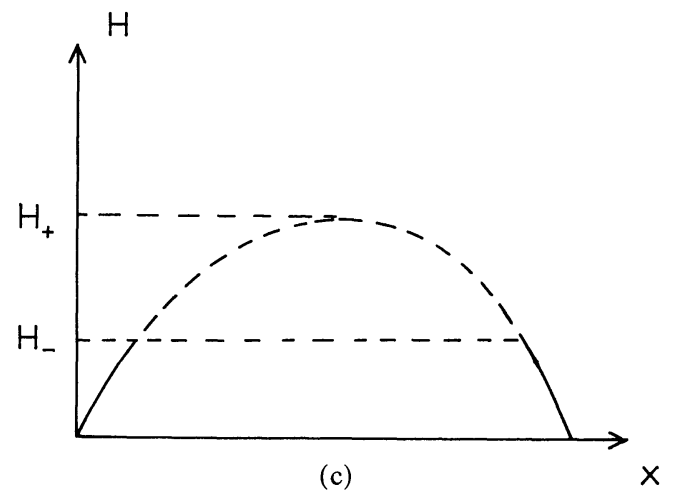

FIG. 5. (a) Pre-surge, quiescent. (b) Activation: rapidly propagating drainage transition fronts. (c) Activated stage. 


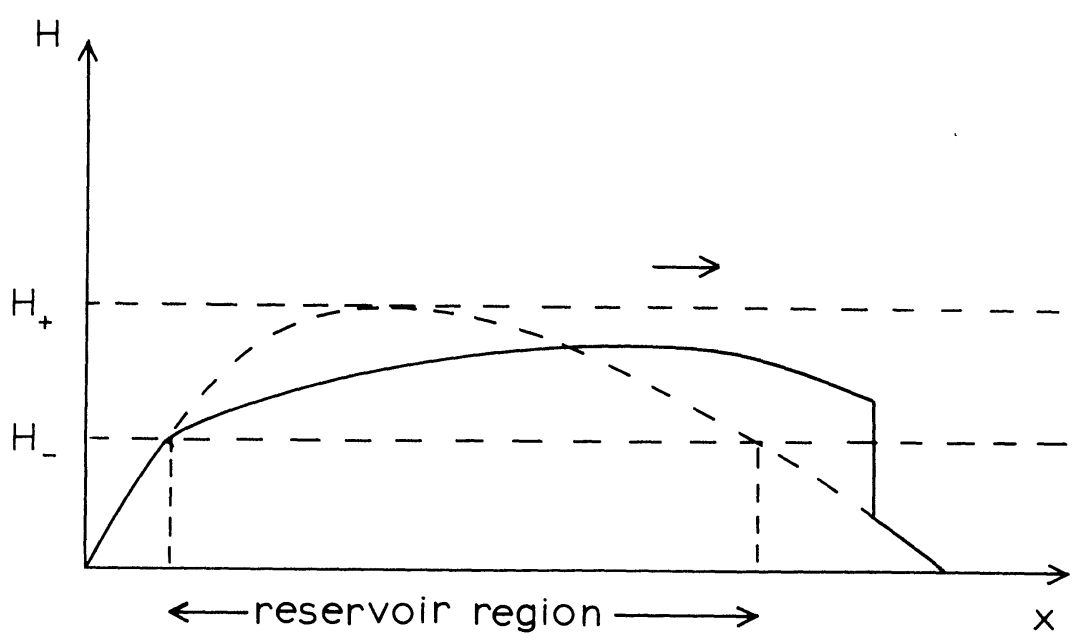

(d)

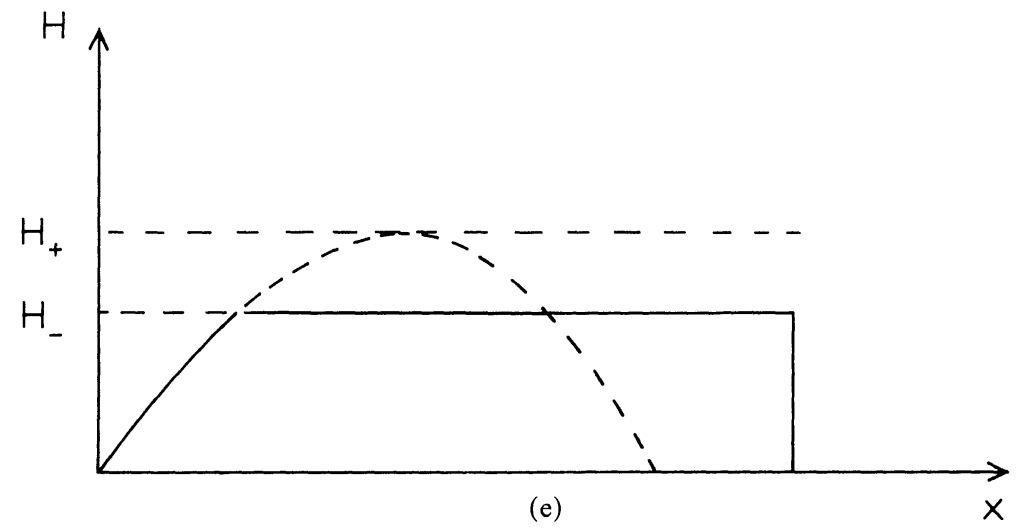

FIG. 5. Continued. (d) Propagation of surge front. (e) Deactivation; post-surge phase.

constant across such a shock, and that the region is thin. To be specific, consider a backwards traveling wave. The equations are (2.1), (3.1), and the drainage law (see (2.2)). By a suitable rescaling, we may choose $N_{R}=1$ and $\Lambda_{c}=1$, so that

$$
\begin{aligned}
g(\Lambda) & =1, & & \Lambda<1, \\
& =\delta, & & \Lambda>1 .
\end{aligned}
$$

We put

$$
H=H^{*}+\varepsilon_{3} \chi, \quad x+V t=\varepsilon_{2} \xi
$$

and expect $V \gg 1, \varepsilon_{2}, \varepsilon_{3} \ll 1$. A leading order balance can be effected in (2.1a) and (2.1b) by choosing

$$
\varepsilon_{3}=1 / V, \quad \varepsilon_{2}=\varepsilon V,
$$

and then we find, to leading order,

$$
\chi_{\xi} \sim-H^{*} u_{\xi},
$$

whence

$$
\begin{gathered}
H^{*}\left(1+\tilde{\mu} u_{\xi}\right)+\tilde{\alpha}\left\{\left|u_{\xi}\right|^{(1 / n)-1} u_{\xi}\right\}_{\xi}=c N^{1-(n / m)} u^{1 / m}, \\
N_{\xi}+N \sim g(\Lambda),
\end{gathered}
$$


where

$$
\tilde{\alpha}=\alpha H^{*} /(\varepsilon V)^{1+(1 / n)}, \quad \tilde{\mu}=\mu H^{*} / \varepsilon V^{2} .
$$

If $x+V t$ is replaced by $x-V t$ in (3.5), the effect is to change the sign of the first derivatives in (3.8). Since for forward traveling waves, the boundary conditions at $\pm \infty$ are reversed, the same model will therefore also describe forward traveling activation waves.

We choose $V$ in order to bring in the largest of the terms $\tilde{\alpha}$ and $\tilde{\mu}$ in (3.8). Taking $\alpha \sim 10^{-2}, \mu \sim 10^{-1}$, and if we guess $\varepsilon V \sim 10^{-1}$, then $\tilde{\alpha}$ (for $n=3$ ) is $\sim 10^{-2 / 3}$, whereas $\tilde{\mu}$ is $\sim 1 / V \sim 10 \varepsilon$. Even for $\varepsilon$ as large as $10^{-2}$ (drainage response time of $\sim$ one month), this is quite moderate; thus we suppose $\tilde{\alpha} \gg \tilde{\mu}$ (the precise value must be found as an eigenvalue from the solution), and will presuppose that $\tilde{\mu} \ll 1$. At leading order, we therefore have

$$
\tilde{\alpha}\left(u_{\xi}^{1 / n}\right\}_{\xi} \sim c N^{1-(n / m)} u^{1 / m}-H^{*},
$$

where we anticipate $u_{\xi}>0$, and where the solution of (3.8b) gives $N$ as

$$
\begin{gathered}
N=1, \quad u<1, \\
N=\left[\delta+(1-\delta) e^{-\xi}\right], \quad u>1 .
\end{gathered}
$$

We wish to solve (3.10), using (3.11), with

$$
\begin{gathered}
u \rightarrow\left(H^{*} / c\right)^{m} \quad \text { as } \xi \rightarrow-\infty, \\
u \rightarrow\left(H^{*} / c\right)^{m} / \delta^{m-n} \quad \text { as } \xi \rightarrow+\infty,
\end{gathered}
$$

and we expect that satisfaction of these boundary conditions requires special choice of $\tilde{\alpha}$.

One of the features of surging glaciers is the large velocity difference between surging and nonsurging states. This corresponds to the assumption that $\delta$ is small in (3.12). With this assumption, an asymptotic solution is possible, in which $\tilde{\alpha}$ is small. To see this, choose the origin so that $\xi=0$ corresponds to the critical value of $u$,

$$
u=u_{c}=1 \text {. }
$$

For $\xi<0$, the solution of

$$
\tilde{\alpha}\left(u_{\xi}^{1 / n}\right)_{\xi}=c u^{1 / m}-H^{*},
$$

with (3.12a) and (3.13) on $\xi=0$, yields

$$
u=u\left[\xi / \tilde{\alpha}^{n /(n+1)}\right], \quad u=1, \quad u_{\xi}=p / \tilde{\alpha}^{n /(n+1)} \quad \text { on } \xi=0,
$$

where $p$ is an $O(1)$ constant, which can be computed. For $\xi>0$, and while $\xi \sim O(1)$, we have $N \sim e^{-\xi}$. We put

$$
u=\tilde{u} / \tilde{\alpha}^{m n /(m-n)},
$$

so that

$$
\left(\tilde{u}_{\xi}^{1 / n}\right)_{\xi} \sim c \tilde{u}^{1 / m} e^{-[1-(n / m)] \xi}+O\left(\delta, \tilde{\alpha}^{n /(m-n)}\right) ;
$$

matching conditions to the solution in $\xi<0$ require

$$
\tilde{u}, \tilde{u}_{\xi} \sim 0 \quad \text { as } \xi \rightarrow 0 .
$$


Neglecting the small terms, the appropriate solution satisfies

$$
\tilde{u} \sim a \xi^{\gamma}, \quad \xi \rightarrow 0,
$$

where

$$
\begin{aligned}
& \gamma=m(n+1) /(m+n)>1, \\
& a=\left[\frac{m c}{\gamma-1}\right]^{m n /(m-n)}\left[\frac{1}{\gamma}\right]^{1 /(m-n)} ;
\end{aligned}
$$

as $\xi \rightarrow \infty$,

$$
\tilde{u} \sim D \xi,
$$

where $D$ can be determined from a numerical solution of (3.17). For $\xi \gg O(1)$, we put

$$
\tilde{u}=U / \nu, \quad \xi=Z / \nu,
$$

and now choose $\nu$ and $\tilde{\alpha}$ so that all three terms in (3.10) balance (note when $\xi \gg 1$, $N \approx \delta$ so long as $\xi$ is algebraically large in $\delta$ ). Specifically, we define

$$
\begin{aligned}
& \nu=c^{m /(m+1)} \delta^{(m-n) /(m+1)}, \\
& \tilde{\alpha}=\left(b / H^{*}\right)^{(m-1) / m} c^{(m-n) /(m+1)} \delta^{(m-n)^{2} / m(m+1)},
\end{aligned}
$$

where $b$ is $O(1)$, to be chosen; then $U$ satisfies, for $Z \sim O(1)$,

$$
\left(U_{Z}^{1 / n}\right)_{Z}=U^{1 / m}-b
$$

with the matching condition from (3.21) giving as initial conditions:

$$
U=0, \quad U_{Z}=D \quad \text { on } Z=0 .
$$

Notice from (3.17) that $D$ can be written as

$$
D=D_{1} c^{m n /(m-n)},
$$

where $D_{1}$ can be computed from (3.17) using $c=1$. We thus integrate (3.24) using (3.25); the extra condition

$$
U \rightarrow b^{m} \text { as } Z \rightarrow \infty
$$

determines the value of $b$, and hence $(3.23 \mathrm{~b})$ gives $\tilde{\alpha}$.

A first integral of (3.24) is

$$
U_{Z}^{(n+1) / n} /(n+1)=m U^{(m+1) / m} /(m+1)-b U+B,
$$

where the constant of integration $B$ satisfies, from (3.25) and (3.27),

$$
B=D^{(n+1) / n} /(n+1)=b^{m+1} /(m+1),
$$

whence

$$
b=\left[\left(\frac{m+1}{n+1}\right) D^{(n+1) / n}\right]^{1 /(m+1)} .
$$

We have $\tilde{\alpha} \sim \delta^{(m-n)^{2} /(m(m+1))}$. For example, if $m=4$ and $n=3$, then $(m-$ $n)^{2} /(m(m+1))=1 / 20$. Thus the dependence on $\delta$ is very weak. We then choose $V$ (the shock speed) from (3.9), that is,

$$
V=\frac{1}{\varepsilon}\left(\alpha H^{*} / \tilde{\alpha}\right)^{n /(n+1)} .
$$


The shock width is really (from (3.6) and (3.22))

$$
\Delta x \sim \varepsilon V / \delta^{(m-n) /(n+1)},
$$

and this is practically $O\left(\alpha^{n /(n+1)}\right)$. For $\alpha=10^{-2}, n=3$, this is $10^{-3 / 2}$. On a $20 \mathrm{~km}$ long glacier, this is about 600 metres, or, in round figures, one kilometre. The velocity of these waves depends on $1 / \varepsilon$. Based on a tunnel collapse time of $\sim$ one day, Fowler [6] suggested $\varepsilon \sim 10^{-5}$, and thus $V \sim 10^{3.5} \approx 3000$, which in terms of a velocity of $0.4 \mathrm{~m}$ day $^{-1}$ is equivalent to 50 metres an hour. This is of the order of magnitude of propagation of mini-surges, which may thus correspond to such pressure waves. However, the unstable transition from tunnel to cavity via leakage to the bed may well occur over a much longer time scale, and until a more complete model than $(2.1 \mathrm{c})$ is available, the appropriate estimate of $\varepsilon$ can only be guessed.

(ii) The surge. After propagation of the activation waves, the activated zone has (neglecting $\alpha, \mu$ and $\varepsilon$ ) velocity given by (3.3), that is,

$$
u=H^{m} /(m+1) \nu
$$

where

$$
\nu=\delta^{m-n} c^{m} /(m+1) \ll 1
$$

We rescale $t$ with $\nu$; thus

$$
t=\nu \tau
$$

so that to leading order

$$
H_{\tau}+H^{m} H_{x} \sim 0
$$

for $x_{-}<x<x_{f}$, where $x_{-}$(constant) is where $H=H_{-}$(upstream), and $x_{f}(\tau)$ is the position of the surge front. The solution can be written implicitly in the form

$$
H=H_{0}\left(x-H^{m} \tau\right),
$$

for $x>x_{-}+H_{-}^{m} \tau$, which is the characteristic emanating from $x_{-}$, and which bounds the disturbance region. A shock forms at $x_{f}$ (see Hutter [9] for further discussion of nonlinear waves on glaciers): the advance of $x_{f}$ is governed by

$$
\frac{d x_{f}}{d \tau} \approx \frac{H^{m+1}\left(x_{f^{-}}, \tau\right)}{(m+1)\left[H\left(x_{f^{-}}, \tau\right)-H_{0}\left(x_{f}\right)\right]},
$$

since the mass flux is $O(\nu)$ in front of the shock. When the front passes the terminus, we have

$$
\frac{d x_{f}}{d \tau} \approx H^{m}\left(x_{f^{-}}, \tau\right) /(m+1),
$$

so that the characteristics behind catch up with the front. However, we see from (3.38) that this is not so initially, and (3.38) would predict infinite shock speed, whereas we require $d x_{f} / d \tau \leqq H_{-}^{m}$, so that characteristics from $x_{f^{-}}$can reach the shock. We surmise that until such time, the front grows unstably, and carries with it the drainage transition region (see below for further discussion). The characteristic diagram is shown in Fig. 6 . We could integrate (3.39) using (3.37) to find $x_{f}(t)$. The duration and extent of the advance can be computed by inspection, however. The total advance of the front is given by $\Delta$, where

$$
H_{-}\left(x_{+}+\Delta-x_{-}\right)=\int_{x_{-}}^{x_{+}+\Delta} H_{0}(\xi) d \xi .
$$




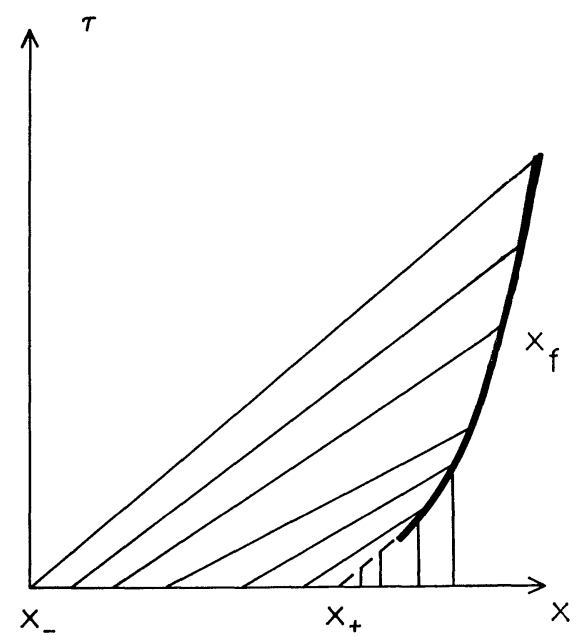

FIG. 6. Characteristic diagram for the solution of (3.36). A shock first forms by intersection of characteristics in $\left(x_{-}, x_{+}\right)$on the (dashed) characteristic from $x_{+}$, at some $\tau=\tau_{c}>0$. However, for $\tau<\tau_{c}$, characteristics from $x_{+}-$intersect that from $x_{+}$. We surmise that there is a transcritical period when the fast incoming activation wave at $x_{+}$, with small depth change, is transferred to a slower wave front advance with large depth change. A very similar analysis appears in Fowler [5]. It is consistent with this description that the activated region first exhibits a bulge (at $\left.x_{f}\right)$, which subsequently propagates as a front. This is consistent with the descriptions of the Variegated surge in phase one and phase two (Kamb et al. [12]).

If we suppose $\Delta$ is quite small, and that $x_{+}$is near the terminus initially, then this is just

$$
\Delta \approx(\overline{\Delta H})\left(x_{+}-x_{-}\right) / H_{-},
$$

where $(\overline{\Delta H})$ is the mean decrease in thickness, approximately $\left(H_{+}-H_{-}\right) / 2$. The duration of the advance is then given by

$$
\begin{aligned}
\tau_{s} & =\left(x_{+}+\Delta-x_{-}\right) / H_{-}^{m} \approx\left(x_{+}-x_{-}\right)\left(H_{-}+(\overline{\Delta H})\right) / H_{-}^{m+1} \\
& \approx\left(x_{+}-x_{-}\right) \bar{H} / H_{-}^{m+1}
\end{aligned}
$$

where $\bar{H}$ is the mean thickness during the surge.

In order to justify the description of the shock front, we need to examine the shock structure. This is conceptually quite similar to the analysis of transcritical shocks by Fowler [5]. We seek a traveling wave solution to (2.1) in the form of functions of $X$, where we put

$$
x-V t=\tilde{\varepsilon} X,
$$

and $\tilde{\varepsilon}$ and $V$ are to be specified (we expect $V$ to be equivalent to (3.38)). Then (neglecting $s^{\prime}(x)$ in $(2.1 \mathrm{a})$, which will be relatively small), we have

$$
\begin{gathered}
H(V-u) \sim \text { constant, } \\
H\left[1-\tilde{\mu} H_{X}\right]+\tilde{\alpha}\left[H u_{X}\left|u_{X}\right|^{(1 / n)-1}\right]_{X}=\left\{\begin{array}{c}
c u^{1 / m}, \\
\delta^{(m-n) / m} c u^{1 / m},
\end{array}\right.
\end{gathered}
$$

where the upper and lower functions apply in front of and behind the drainage transition region, respectively. Here

$$
\tilde{\alpha}=\alpha / \tilde{\varepsilon}^{1+(1 / n)}, \quad \tilde{\mu}=\mu / \tilde{\varepsilon}
$$


Notice that $\tilde{\alpha}, \tilde{\mu}$ and $V$ are defined differently than in (i). We can locate the drainage transition at $X=0$ without loss of generality. For $X<0$, we put

$$
u=U / \delta^{m-n}
$$

and will expect boundary conditions $U, U_{X} \sim 0$ at $X=0$. We put

$$
V=\tilde{V} / \delta^{m-n}
$$

thus (if $\mathrm{H}^{+}$and $\mathrm{H}^{-}$are the values of $H$ downstream and upstream of the shock) (3.44a) implies

$$
\tilde{V} \sim H^{-} U^{-} /\left(H^{-}-H^{+}\right), \quad U^{-}=\left(H^{-} / c\right)^{m}
$$

It follows that

$$
H(\tilde{V}-U) \sim H^{+} \tilde{V}=C, \text { say, }
$$

and $(3.44 \mathrm{~b})$ is

$$
H\left[1-\tilde{\mu} H_{X}\right]+\left(\tilde{\alpha} / \delta^{(m / n)-1}\right)\left[H U_{X}\left|U_{X}\right|^{(1 / n)-1}\right]_{X} \sim c U^{1 / m} .
$$

We must choose the dimensionless shock width $\tilde{\varepsilon}$ so that one or the other derivative term in (3.50) is important. Let us choose $\tilde{\alpha}=\delta^{(m / n)-1}$, that is,

$$
\tilde{\varepsilon}=\left[\alpha^{n} / \delta^{m-n}\right]^{1 /(n+1)} ;
$$

a typical value, for $n=3, \alpha=10^{-2}, \delta^{m-n}=10^{-2}$ (a hundred fold increase of velocity), is $\tilde{\varepsilon} \sim 10^{-1}$, corresponding to two kilometres on a $20 \mathrm{~km}$ length glacier. Then also $\tilde{\mu} \sim O(1)$, so that we can expect both terms to be important. Thus the problem is to solve

$$
\begin{aligned}
& H\left(1-\tilde{\mu} H_{X}\right)+\left[H U_{X}\left|U_{X}\right|^{(1 / n)-1}\right]_{X} \sim c U^{1 / m}, \\
& H=C /(\tilde{V}-U),
\end{aligned}
$$

in $X<0$, with

$$
\begin{aligned}
& u \rightarrow U^{-} \text {as } X \rightarrow-\infty, \\
& U=U_{X}=0 \quad \text { on } X=0 .
\end{aligned}
$$

The problem in $X>0$ can be simplified somewhat, since $H=H^{+}+O\left(\delta^{m-n}\right), X \sim$ $\delta^{(m-n) /(n+1)}, U \sim \delta^{m-n}$ there; however, we do not pursue this further (see Fowler [5] for a similar analysis).

Equation (3.52) must be solved numerically, by picking $C$ and $\tilde{V}$ and integrating backwards towards $-\infty$. $U_{-}$and hence $H^{-}$are then determined in terms of $\tilde{V}$ and $H^{+}$. For large values of $-X$, let $U=U^{-}+v$. Approximating (3.52) for small $v$, we find

$$
\left[v_{X}\left|v_{X}\right|^{(1 / n)-1}\right]_{X}-\beta v_{X}+\gamma v \simeq 0
$$

where

$$
\beta=\tilde{\mu} H_{-}^{2} / C, \quad \gamma=\left(m H_{-} U_{-}-C\right) / m U_{-} C .
$$

It is not difficult to see that $v$ necessarily tends to zero as $X$ tends to $-\infty$, only if $\gamma>0$ (consider the "energy" relation $d E / d(-X)=-\beta v_{X}^{2}$, where $E=\left|v_{X}\right|^{(n+1) / n} /(n+1)+$ $\gamma v^{2} / 2$ ), whereas if $\gamma<0$, then $v=0$ is a saddle point, which suggests that (3.52) cannot be satisfied with both boundary conditions at $X=0$. We therefore expect solutions to exist to (3.52) with (3.53), providing $\gamma>0$, that is, using (3.48) and (3.49),

$$
H_{-}-H_{+}>H_{+} / m
$$


and in this case the decay as $X \rightarrow-\infty$ of the velocity profile will be oscillatory when $\gamma>\beta^{2} / 4$, for the particular case $n=1$, that is, if

$$
H_{-}-H_{+}>H_{+} / m+\tilde{\mu}^{2} H_{-}^{2}\left(H_{-}-H_{+}\right)^{2} / 4 H_{+} H_{-} .
$$

In practice (e.g., $\tilde{\mu}=1, H_{+}=1, U_{-}=1, m=4$ ), oscillatory decay can be expected, and is indeed observed (Kamb et al. [12, Fig. 4]). For $n>1$, it is more likely, since when $v \ll 1$, then the energy $E \sim v^{2}$, so $\left|v_{X}\right| \sim v^{2 n /(n+1)}$, and $d E / d(-X) \sim v_{X}^{2} \sim v^{4 n /(n+1)} \ll E$, so that an oscillatory decay is assured.

The restrictive criterion for the existence of the waves discussed here, (3.56), is exactly equivalent to the condition that characteristics from $x_{f^{-}}$reach the shock, and implies that some other analysis is necessary in the initial stages of the surge, $0<$ $H_{-}-H_{+}<H_{+} / m$. This is not too surprising, since the arriving activation wave has width $O\left[\alpha^{n /(n+1)} \delta^{-(m-n)^{3} / m(m+1)(n+1)}\right]$ and speed $O\left[\alpha^{n /(n+1)} \delta^{-n(m-n)^{2} / m(m+1)(n+1)} \varepsilon^{-1}\right]$, whereas the surge front has width $O\left[\alpha^{n /(n+1)} \delta^{-(m-n) /(n+1)}\right]$ and speed $O\left(\delta^{-(m-n)}\right)$. We surmise that the transition between these two regimes occurs in a transcritical regime, where both the change in drainage and jump in depth occur over the same length scale. A mathematically similar problem was considered by Fowler [5], but we do not attempt to pursue the analysis here. The physical suggestion is that in the initial stages of a surge, a surge front grows in height unstably until it satisfies (3.56) (which it must eventually do).

(iii) Deactivation wave. It can be seen from Fig. 6 that the active surge phase is terminated by the passage of a characteristic from $x_{-}$, which propagates with speed (from (3.37)) $d x / d t=O\left(\delta^{-(m-n)}\right)$. It is reasonable to suppose that a hydraulic deactivation wave accompanies this characteristic. We write

$$
\begin{aligned}
& x-\left(\hat{V} / \delta^{m-n}\right) t=\hat{\varepsilon} X, \\
& H=H_{-}+\hat{\varepsilon} \chi \\
& u=U / \delta^{m-n}
\end{aligned}
$$

so that to leading order $(2.1 \mathrm{c})$ becomes

$$
-\hat{V}\left(\varepsilon / \delta^{m-n} \hat{\varepsilon}\right) N_{X}+N=g(\Lambda) .
$$

We anticipate that $\hat{\varepsilon} \gg \varepsilon / \delta^{m-n}$, so that the hydraulic switch occurs on a length scale $X \ll 1$, and $f(\Lambda)$ is then given by (cf. (3.44))

$$
f= \begin{cases}c u^{1 / m}, & X<0, \\ \delta^{(m-n) / m} c u^{1 / m}, & X>0,\end{cases}
$$

where we may take the switch at $X=0$ without loss of generality. To leading order, (2.1) then gives

$$
\begin{aligned}
& \chi \sim H_{-} U / \hat{V}, \\
& H_{-}+H_{-}\left[U_{X}\left|U_{X}\right|^{(1 / n)-1}\right]_{X} \sim c U^{1 / m},
\end{aligned}
$$

for $X>0$, bearing in mind $\mu \ll 1$, and putting

$$
\hat{\varepsilon}=\left[\alpha \delta^{-(m-n) / n}\right]^{n /(n+1)} .
$$

Our assumption that $N$ switches on a scale $X \ll 1$ is then tantamount to assuming

$$
\varepsilon \ll \delta^{m-n} \hat{\varepsilon}=\left[\alpha^{n} \delta^{(m-n)}\right]^{1 /(n+1)} .
$$


If $\delta^{m-n}=10^{-2}, \alpha=10^{-2}$, and $n=3$, this is $\varepsilon \ll 10^{-2}$, which is feasible. The boundary conditions for $U$ are

$$
\begin{aligned}
& U=0 \quad \text { on } X=0, \\
& U \rightarrow U_{\infty}=\left(H_{-} / c\right)^{m} \text { as } X \rightarrow \infty,
\end{aligned}
$$

and the flow behind uncouples. Equation (3.62) may be written as

$$
\left(U_{X}^{1 / n}\right)_{X}=\left(U / U_{\infty}\right)^{1 / m}-1,
$$

from which $U_{X}=O(1)$ at $X=0$ may be found. In order to satisfy continuity of $u$ and $u_{X}$ at $X=0$, the outer solution for $u$ in $X<0$ of

$$
H_{-}-\mu H_{-}^{2} u_{X}+\delta^{m-n} H_{-}\left[u_{X}\left|u_{X}\right|^{(1 / n)-1}\right]_{X} \sim c u^{1 / m},
$$

that is, $u \sim U_{-}=\left(H_{-} / c\right)^{m}$, must be modulated by a boundary layer near $X=0$. We do not pursue the details. Again, comparison can be made with Fowler [5].

4. Discussion. The model equations (2.1) which we have used in describing surge phenomena are based on real physics, but are nevertheless not necessarily quantitatively accurate. The primary purpose of this paper is to suggest that a rough overall description of the flow as a spatially-varying relaxation oscillator, as portrayed in Fig. 5, can be mathematically validated by detailed analysis of the connecting traveling-wave structures. It remains to consider the possibility of making testable predictions (e.g., surge criteria) using this model. As we shall see, this is not likely to be a very profitable exercise.

There are two sets of predictions we would like to make. First, what parametric criterion distinguishes surging from nonsurging glaciers? Although there are various side issues, the principal criterion is that in a positive steady state, drainage transition should be able to take place, that is, $\Lambda>\Lambda_{c}$ (see the discussion preceding (2.2)). In order to interpret this in terms of physical parameters, we use the following definitions (Fowler [6], [7], and where for comparison we do not rescale $\Lambda$ with $\Lambda_{c}$, as in (3.4)):

$$
\begin{aligned}
& \tau \sim c_{1} d \tilde{\chi}, \\
& N \sim c_{2} \tilde{\chi}^{11 / 8 n} \tilde{q}^{1 / 4 n}, \\
& u d \sim \Phi, \\
& \tau \sim c_{4} N^{1-n / m} u^{1 / m}, \\
& \Lambda \sim c_{5} u / N^{n}, \\
& \Lambda_{c} \sim c_{6} \tilde{\chi}^{-9 m /\{2(m+2)\}} N^{3 m n /(m+2)} .
\end{aligned}
$$

The dimensional variables are $\tau$ (shear stress), $N$ (effective pressure), $u$ (longitudinal velocity), and $d$ (depth); $\Lambda$ and $\Lambda_{\mathrm{c}}$ have the same meaning as previously. $\tilde{\chi}$ and $\tilde{q}$ are scaled values of mean bedrock slope $\chi$ and subglacial drainage $q$, where we have put

$$
\tilde{\chi}=10 \chi, \quad \tilde{q}=q /\left[10 \mathrm{~m}^{3} \mathrm{~s}^{-1}\right],
$$

so that typically $\tilde{\chi}, \tilde{q} \sim 1$. The flux $\Phi$ is a measure of climatic accumulation, typically in the range $10^{4}-10^{5} \mathrm{~m}^{2} \mathrm{y}^{-1}$. The different relations in (4.1) have the following meaning: (4.1a) is the shear stress balance, $(4.1 \mathrm{~b})$ is the approximate steady tunnel drainage characteristic, (4.1c) is conservation of mass, (4.1d) is the sliding law, (4.1e) and (4.1f) reflect the stability characteristics of tunnel drainage. Estimates for the parameters in 
(4.1) that can be made, so as to be consistent with observations, particularly of Variegated Glacier (Kamb et al. [12], Bindschadler [1]) are as follows:

$$
\begin{aligned}
c_{1} & \sim 10^{-2} \mathrm{bar} \mathrm{m}^{-1}, \\
c_{2} & \sim 10 \mathrm{bar}, \\
c_{4} & \sim 10^{-1+n / m} \mathrm{bar}^{n / m} \mathrm{~m}^{-1 / m} \mathrm{y}^{1 / m}, \\
c_{5} & \sim 10^{n-2} \mathrm{bar}^{n} \mathrm{~m}^{-1} \mathrm{y}, \\
c_{6} & \sim 10^{-3 m n /(m+2)} \mathrm{bar}^{-3 m n /(m+2)},
\end{aligned}
$$

and we find that the surge criterion $\Lambda>\Lambda_{c}$ reduces to

$$
\Phi \geq 10^{4+2 / m} \tilde{\chi}^{[(11 / 8 n)+3 /\{8(m+2)\}]} \tilde{q}^{[1+(1 / 4 n)-3 /\{4(m+1)\}]} \mathrm{m}^{2} \mathrm{y}^{-1} .
$$

Now in reality, we would expect the discharge $\tilde{q}$ to depend on $\Phi$ via the width $W$; thus we put

$$
\tilde{q}=\tilde{W}\left[\Phi /\left(10^{5} \mathrm{~m}^{2} \mathrm{y}^{-1}\right)\right]
$$

where the scaled width $\tilde{W}=W /(1 \mathrm{~km})$ (so $\tilde{W} \sim 1$ for Variegated Glacier). Approximately, (4.4) is then

$$
\Phi \geq 10^{5} \tilde{\chi}^{p} \tilde{W}^{q} \mathrm{~m}^{2} \mathrm{y}^{-1}
$$

where

$$
p=\frac{11(m+2)+3 n}{2(3 n-m-2)}, \quad q=\frac{4 n(m+2)}{3 n-m-2}-1 .
$$

For values $m=4, n=3$, we find $p \approx 12, q \approx 23$. Consequently, (4.6) is essentially (when $p, q>0)$

$$
\tilde{\chi} \tilde{W}^{\beta} \lesssim O(1), \quad \beta=q / p \approx 2,
$$

if $\Phi \sim 10^{5} \mathrm{~m}^{2} \mathrm{y}^{-1}$, and is essentially independent of $\Phi$.

There are two main comparisons to be made to this result. The first is to Budd's [2] surging criterion, which is

$$
\Phi \tilde{\chi} \geq O\left(10^{5} \mathrm{~m}^{2} \mathrm{y}^{-1}\right),
$$

and evidently completely different. Budd's data for surging glaciers suggests $\ln [\Phi \tilde{\chi}] \approx$ constant for the seven examples he chooses. His data, or that of Meier and Post [17], is also consistent with (4.8), with approximate equality for $\beta \approx 0.8$. In this connection, notice that use of Bindschadler's [1] values $m=3, n=2$ for Variegated Glacier give $\beta \approx 1.3$.

The second comparison is to the statistical analysis of Clarke et al. [4]. Their conclusion was that surging behaviour correlated with length, but not (independently) with surface slope. However, since long glaciers are also less steep, this could equally well be interpreted as meaning that gently-sloping glaciers tend to surge, consistently with (4.8), but in contradiction to (4.9). Clarke et al. [4] did not report the widths of the glacier in their data set. It would be very interesting to test for the validity of a criterion such as (4.8), which involves purely geometrical criteria. 
The second set of predictions which we could make from this model are those concerning depth reduction, period, advance, and duration of surges. From the results of this paper, we could estimate the dimensional values of these as follows:

$$
\begin{array}{ll}
\text { depth reduction: } & \Delta d=d_{+}-d_{-} \sim d \Delta H, \\
\text { period: } & P \sim \Delta d / V, \\
\text { advance: } & \Delta l \sim l\left(d_{+}-d_{-}\right)^{2} / d_{+} d_{-}, \\
\text {duration: } & \Delta t \sim \Delta l / u_{+},
\end{array}
$$

where $d_{+}, d_{-}$are pre- and post-surge depths, and $u_{+}$is surge velocity. A reasonable variety of these quantities can occur (Meier and Post [17]), but there is a problem in using the present results. Using (3.3), we would predict

$$
H_{-} / H_{+} \sim \delta, \quad u_{+} / u_{-} \sim 1 / \delta^{m-n},
$$

neither of which is consonant with the data from Variegated Glacier, which would suggest $\delta \approx \frac{1}{4}, u_{+} / u_{-} \sim 100$, and $H_{-} / H_{+} \sim \frac{3}{4}$. It is specifically the large drop in depth which cannot be reconciled with observation. Indeed, (4.10c) suggests that typically $\Delta d / d \ll 1$ (since $\Delta l / l \ll 1$ is observed). We are forced to conclude that, whereas the description of the mechanics of the flow may be sound, the detailed forms of sliding law and drainage transition criterion in (3.3) cannot be used to make specific predictions.

There are a whole host of reasons why this may be so. Most obvious is the neglect in our analysis of transverse variations in the flow. Glaciers are of finite width, and they flow in valleys of nonhorizontal cross sections. Therefore depth, overburden pressure, and effective pressure will all vary across the valley width. This variation is often taken account of by a "shape factor" (Paterson [19]), which, for example, is used in computing the mean shear stress. However, it is more complicated to take account of the effect of lateral variations in $N$ and $H$, when a switch in drainage occurs.

Apart from this, we may question the quantitative validity of the two fundamental features of the model, i.e., the sliding law $\tau \propto N^{a} u^{b}$, and the drainage switching criterion $u>c N^{n}$. Both of these prescriptions rely on a detailed sub-model of conditions at the glacier bed, and whereas there is some agreement on the qualitative form, the quantitative details must be considered uncertain. The sliding law is based on a theoretical consideration of lubricated flow over a rough bedrock (Fowler [7]), whereas it is thought that many glaciers actually slide at their bases on layers of deformable debris (glacial till), which is eroded from the underlying bedrock. A notable example is Trapridge Glacier (Clarke et al. [3]). Although in this case the form of the sliding law assumed here may be appropriate (cf. Bindschadler [1]), there is no obvious justification for supposing that the exponents, or coefficients, are the same. In particular, the drainage switching criterion is likely to be different. Even within the context of the present model, Walder [22] and Kamb [13] have given two radically different criteria for drainage transition: $N<$ constant (Walder), or $\mathrm{Nu}<$ constant (Kamb).

Our conclusion must be that, whereas the qualitative mathematical description given in this paper may be appropriate, nevertheless the detailed prediction of such features as surge duration and advance, etc., must await more conclusive studies of the sliding and drainage processes at the bed.

5. Conclusions. Despite the caveats advanced in the preceding section, the model proposed here is realistic in the sense that it is based on reasonable physics. The description of basal sliding and drainage is consistent with observations on Variegated Glacier. Given this model, we have shown how it will predict surging to occur if the 
drainage switching criterion is satisfied, and we have shown how a surge consists of fast and slow flow phases, separated by activation and deactivation waves, where the hydraulic drainage system is altered. There are further qualitative tests to which this model may be subjected. It should be able to explain seasonal shut-down of surges, seasonal waves (Hodge [8]), by including a seasonal variation in $N$, and it should be able to explain the propagation of "mini-surges" (Kamb and Engelhardt [14]) analogously to wave propagation in excitable media, for values of $H$ such that $0<H_{+}-H \ll 1$. Analyses of such phenomena are reserved for future work.

\section{REFERENCES}

[1] R. BINDSCHADLER, The importance of pressurised subglacial water in separation and sliding at the glacier bed, J. Glaciol., 29 (1983), pp. 3-19.

[2] W. F. BUDD, A first simple model for periodically self-surging glaciers, J. Glaciol., 14 (1975), pp. 3-21.

[3] G. K. C. Clarke, S. G. Collins, And D. E. Thompson, Flow, thermal structure, and subglacial conditions of a surge-type glacier, Canad. J. Earth Sci., 21 (1984), pp. 232-240.

[4] G. K. C. Clarke, J. P. Schmok, C. S. L. Ommanney, and S. G. Collins, Characteristics of surge-type glaciers, J. Geophys. Res., 91 (1986), pp. 7165-7180.

[5] A. C. Fowler, Waves on glaciers, J. Fluid Mech., 120 (1982), pp. 283-321.

[6] —, A theory of glacier surges, J. Geophys. Res., 92 (1987), pp. 9111-9120.

[7] - Sliding with cavity formation, J. Glaciol., (1987), J. Glaciol., pp. 255-267.

[8] S. M. Hodge, Variations in the sliding of a temperate glacier, J. Glaciol., 13 (1974), pp. 349-369.

[9] K. HutTER, Theoretical Glaciology, Reidel, Dordrecht, 1983.

[10] A. IKEN, The effect of the subglacial water pressure on the sliding velocity of a glacier in an idealized numerical model, J. Glaciol., 27 (1981), pp. 407-421.

[11] A. IKEN AND R. BINDSCHADLER, Combined measurements of subglacial water pressure and surface velocity of the Findelengletscher, Switzerland. Conclusions drawn on drainage system and sliding mechanism, J. Glaciol., 32 (1986), pp. 101-119.

[12] B. Kamb, C. F. Raymond, W. D. Harrison, H. Engelhardt, K. A. Echelmeyer, N. Humphrey, M. M. BRugman, AND T. PFefFer, Glacier surge mechanism: 1982-1983 surge of Variegated Glacier, Alaska, Science, 227 (1985), pp. 469-479.

[13] В. КАМв, Glacier surge mechanism based on linked cavity configuration of the basal water conduit system, J. Geophys. Res., 92 (1987), pp. 9083-9100.

[14] B. KAMB AND H. ENGELHARDT, Waves of accelerated motion in a glacier approaching surge: the mini-surges of Variegated Glacier, Alaska, USA, J. Glaciol., 33 (1987), pp. 27-46.

[15] L. A. Lliboutry, General theory of subglacial cavitation and sliding of temperate glaciers, J. Glaciol., 7 (1968), pp. 21-58.

[16] - Local friction laws for glaciers: a critical review and new openings, J. Glaciol., 23 (1979), pp. 67-95.

[17] M. F. MEIER AND A. S. Post, What are glacier surges? Canad. J. Earth Sci., 6 (1969), pp. 807-817.

[18] J. F. NYE, A calculation on the sliding of ice over a wavy surface using a Newtonian viscous approximation, Proc. Roy. Soc. London Ser. A, 311 (1969), pp. 445-467.

[19] W. S. B. PAterson, The Physics of Glaciers, Pergamon, Oxford, 1981.

[20] - The current state of research on hydraulic effects at the glacier bed. An introduction to the workshop, in Hydraulic Effects at the Glacier Bed and Related Phenomena, Mitteilungen der Versuchsanstalt fur Wasserbau, Hydrologie und Glaziologie, Nr. 90, ETH, Zürich, 1986, pp. 7-14.

[21] H. Röthlisberger, Water pressure in intra- and subglacial channels, J. Glaciol., 11 (1972), pp. 177-203.

[22] J. S. WALDER, Hydraulics of subglacial cavities, J. Glaciol., 32 (1986), pp. 439-445. 